\title{
Design and Analysis of a Controlled Diffusion Aerofoil Section for an Axial Compressor Stator and Effect of Incidence Angle and Mach No. on Performance of CDA
}

\author{
Nilesh P. Salunke ${ }^{1}$, S. A. Channiwala ${ }^{2}$ \\ ${ }^{1}$ Head Department of Mechanical Engineering, \\ R. C. Patel Institute of Technology, Shirpur. 425405 INDIA \\ ${ }^{2}$ S.V.N.I.T. Surat, Icchanath, Surat
}

\begin{abstract}
This paper deals with the Design and Analysis of a Controlled Diffusion Aerofoil (CDA) Blade Section for an Axial Compressor Stator and Effect of incidence angle and Mach No. on Performance of CDA. CD blade section has been designed at Axial Flow Compressor Research Lab, Propulsion Division of National Aerospace Laboratories (NAL), Bangalore, as per geometric procedure specified in the U.S. patent (4). The CFD analysis has been performed by a 2-D Euler code (Denton's code), which gives surface Mach No. distribution on the profiles. Boundary layer computations were performed by a 2-D boundary layer code (NALSOF0801) available in the SOFFTS library of NAL. The effect of variation of Mach no. was performed using fluent. The surface Mach no. distribution on the CD profile clearly indicates lower peak Mach no. than MCA profile. Further, boundary layer parameters on CD aerofoil at respective incidences have lower values than corresponding MCA blade profile. Total pressure loss on CD aerofoil for the same incidence range is lower than MCA blade profile.
\end{abstract}

Keywords: CDA, MCA, Blade Profile, Transonic compressor design, Axial Flow Compressor,

\section{Introduction}

The standard series of blades such as the NACA65/Circular Arc, or Double Circular Arc, NACA 400, Multipe Circular Arc Aerofoil, have been used in most of the current production compressors. A new compressor blade type is discussed here which has been developed as a replacement to the contemporary series. Controlled Diffusion Aerofoil is designed and optimized specifically for subsonic and transonic cascade applications. Through control of the diffusion of the aerofoil section, significant boundary layer separation can be avoided over the entire range of aerofoil operation for transonic application. Diffusion from supersonic to subsonic local surface velocity can be accomplished without developing shock waves. A family of airfoils capable of achieving the conditions has been developed which can be described by a number of geometric parameters. Computational Analysis clearly indicated an achievement of low loss and increased incidence range at elevated Mach. Number. A comparatively high loading level, thicker leading and trailing edges can be achieved without affecting performance. These attributes can be utilized to achieve high compressor efficiency, fewer compressor blades and vanes, improved stability, improved durability and reduced development cost.

\section{Background}

The abrupt increase in the loss and decrease in turning of cascade compressor airfoils, as incident Mach number increases, is analogous to the transonic drag rise experienced by isolated airfoil section. As Mach number increases local patches of supersonic flow appear on airfoil surfaces, usually terminated by shock waves. The total pressure loss of the shock plus losses caused by shock induced boundary layer separation combine to limit the low loss Mach number range of cascade airfoils. To improve compressor efficiency, airfoil sections usually have been selected having thinner leading edges at high Mach numbers, in order to increase the transonic drag rise Mach number. However, this section results in decreased useful incidence angle range and decreased durability.

Received September 13 2009; accepted for publication October 14 2009: Review conducted by Prof. Wang Le-qing. (Paper number O09042)

Corresponding author: S. A. Channiwala, Professor, sac@med.svnit.ac.in 
Whitcomb et al.[1] in 1965, demonstrated experimentally the existence of shockless

supercritical flows for isolated airfoil sections. Bauer, Garbedian and Kahn [ 2,3 ] provided analytical design procedure for supercritical wing sections which, through solution of potential equation in complex plane, permitted an airfoil shape to be derived from a specified shockless surface Mach number distribution. The Design and Analysis of a Controlled Diffusion Aerofoil (CDA) Blade Section for an Axial Compressor Stator and Comparison with a Conventional Multiple Circular Arc (MCA) Profile is presented here. This design approach of CDA conforms to the U.S patent specification by Lubenstein, et al[4].

\section{Different Types of Blade Profile}

The two main classes of blades shapes are DCA and MCA. The DCA blade shape is used for high subsonic to low supersonic Mach numbers. The MCA blade shape is used extensively at higher supersonic relative Mach numbers. The polynomial (arbitrary) shape allows more flexibility than the MCA shape and in fact can duplicate the DCA and MCA shapes through proper selection of the polynomial coefficients. The polynomial shape can be used to approximate the various airfoil series as mentioned above and also a new generation of aerofoils, called the Controlled Diffusion Aerofoils (CDA) shape.

CDA shape is the one that employs the concept of shaping the blade beyond the point of peak suction of the surface velocity such that the diffusion rate and associated suction boundary layer results in minimum loss for the airfoil section. This is being employed with high degree of success for moderate to high subsonic and even to transonic blade elements. The supercritical blade shape is a special class of CDA's where in addition to controlling diffusion beyond peak suction surface velocity, the forward portion of the blade is given a shape to perform supercritical operation.

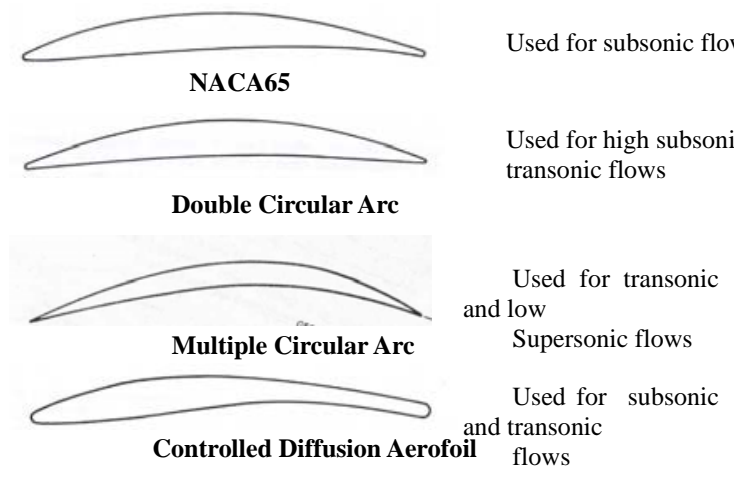

Fig. 1 Different blade profiles
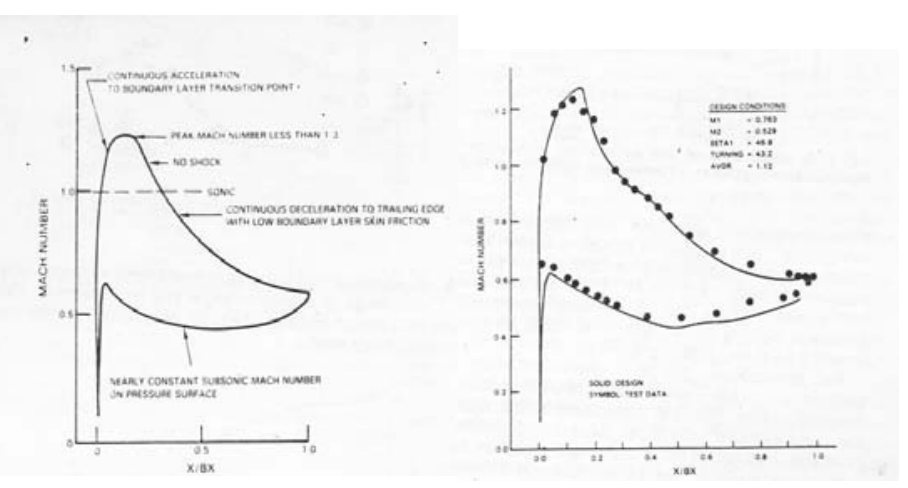

Fig. 2 Comparison of Mach No. distribution on CDA and MCA

\section{Typical Characteristics of CDA and Comparison with MCA}

The salient features of CDA as shown in Fig.2 are as follows

A continuous acceleration from the leading edge to the peak Mach number (at about 20\% chord) on the aerofoil suction surface, to avoid premature laminar boundary layers separation or transition.

A peak Mach number less than 1.3 to avoid boundary layer separation, which could be induced by severe shock wave boundary layer interaction should a shock wave develop at off design conditions.

A continuous shock free deceleration from the peak to suction surface Mach No. to the trailing edge, maintaining a turbulent boundary layer with a low level of skin friction and avoiding separation ahead of the trailing edge.

A nearly constant subsonic Mach number distribution on the pressure surface.

In comparison, at first look the Mach number distribution of CDA and MCA appears to be similar. The peak Mach number on the suction surface for MCA is higher than that of CDA. In case of NACA65 and DCA profile the peak suction surface Mach number occurs at $40 \%$ to $50 \%$ of the blade chord. Thus the blade loading is shifted within the forward half of the chord about $20 \%$. In case of CDA the rear half is more or less straight thereby enabling smooth and continuous deceleration up to trailing edge.

\section{Base Line Parameter}

$$
\mathrm{t} / \mathrm{c}=0.05397, \quad \mathrm{M}_{1}=0.75, \beta 1=46.947, \sigma=1.955 \text {. }
$$

\section{Design Methodology for CDA Profile}

The design procedure involves generation of CD aerofoil for the given flow turning and geometric constraints. The design procedure has following four steps As given in the Lubestein et al (4) U.S patent.

Step 1: -Constuction of mean camber line.

Step2: - Establishing thickness distribution over chord.

Step 3: - Fitting thickness distribution on mean camber line.

Step 4: - Closure of leading and trailing edge. 
Using this procedure a code has been developed in FORTRAN by Dr. B. R. Pai, M. Anbarasu (9). The validity of this code in generating reasonably good CDA sections has been shown by Nagpurwala et al $(6,7)$. However the code suffers from certain inherent deficiencies. Firstly in the calculation of final deviation angle, a slight change in incident angle resulted in an abnormally large change in outlet metal angle. Secondly the iteration procedure for constructing the rear circular arc was not comprehensive enough. The programme did work well within certain incidence range but the blade shape could not be totally relied upon. A carefully line by line debugging of the computer programme was carried out to overcome the shortcomings and modifications were made in the code to give correct deviation angle with respect to incidence angle. The iteration procedure was worked upon to meet all the conditions of tangency given in the patent.

\section{Profile Optimisation}

The optimisation of CD aerofoil should be based on its aerodynamic performance including surface Mach number distribution, total pressure loss coefficient, diffusion factor etc. The optimisation can be effected by varing parameter like maximum thickness, its location, leading and trailing end shapes, location of Tp and incidence angle e.t.c.

A 2-D viscous code for flow computation is absolutely essential for better results but the time consumed is enormous. A quick optimisation approach has been adopted by using a 2-D Euler code (Denton code), which gives fast results .The optimization is based on varying the incidence as a design variable and selecting a profile by studying the surface Mach number distribution .The selected CDA profile is then further analysed for off design incidences, boundary layer behavior and total pressure loss by 2-D Euler code and boundary layer code.

\section{CFD Analysis}

In the CFD analysis of original MCA as well as CDA the following codes were used

2-D Euler code (Denton's code)

NAL-CFO801 Boundary code

Fluent

Incidence angle was chosen as the optimizing parameter for design of CDA blade section. Five different CDA profiles were generated with the above method for incidence angles of $+2,0,-2,-4,-4.947 \mathrm{deg}$. The corresponding inlet metal angles were 40, 42,44,46,46.947 deg. respectively. The five different CDA profiles are shown in Fig. 3. The original MCA profile is also plotted for comparison. The CDA profiles differ from the conventional MCA profile with respect to the maximum thickness which occurs towards the forward portion with leading edge having an elliptical shape and also features a thicker aft portion. It is also observed that with the increase in metal angle, the higher camber angle is accommodated in the forward region, leading to an increased forward loading of the blade. Also the CDA profiles have lower stagger angle than MCA and the stagger angle reduces with increase in incidence angle from negative to positive value. After obtaining different CDA profiles at different incidence angles the next task was to select one of them, which would give better aerodynamic characteristics than the original MCA profile. An ideal approach is to straight away estimate the pressure loss across each profile with the help of a suitable viscous code. But this would be time consuming. Hence it was decided to select a profile based on the surface Mach number distribution, which could be quickly obtained by running the 2-D Denton's code. Coarser grid was selected because during the course of earlier works it had produced close match between experimental and computational results and computational speed was also faster for further analysis through Denton's code.

After selecting the grid size and all other input parameters, the results of the Denton's code in the form of Mach number distribution on different CDA designs for incidence angles ranging from +2 to -4.947 are as shown in Fig. 4 . It is observed that the peak Mach number for -2 deg design incidence angle is 0.98 , which is lower than that for all other CDA designs and much lower than that for the original profile (peak $M=1.1$ ). As the design incidence angle increases towards negative side from $\mathrm{i}=-2$, peak suction Mach number and exit Mach number increases whereas exit static pressure decreases and with increase in incidence towards positive side from $\mathrm{i}=-2$, the peak Mach number again increases with shift towards leading end at 8 to $10 \%$ of the chord

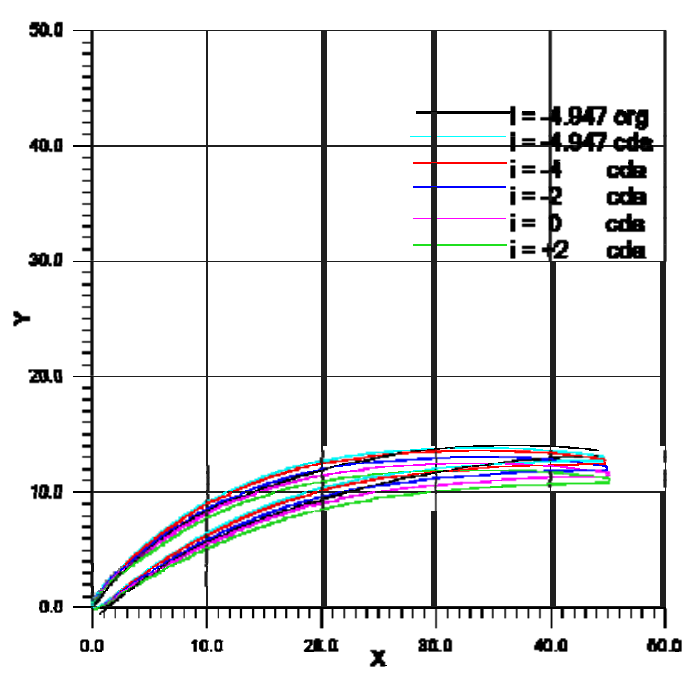

Fig. 3 Comparison of Different CDA and MCA

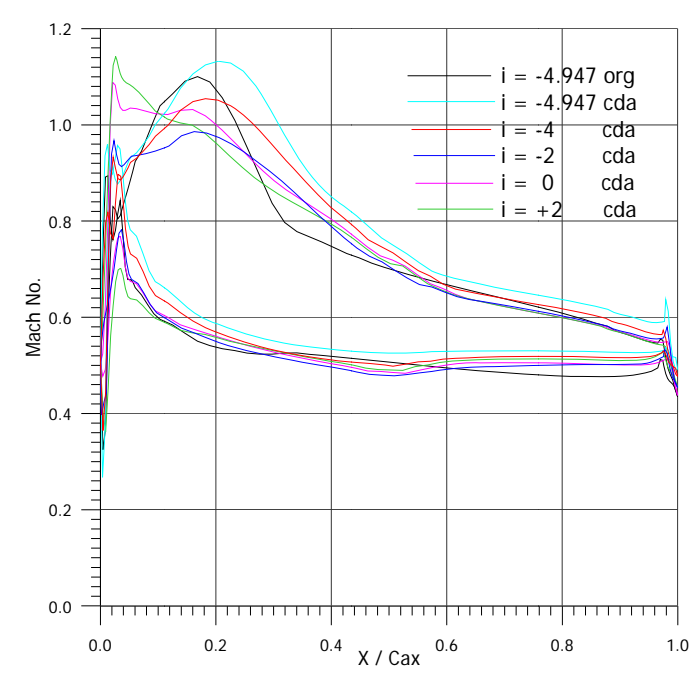

Fig. 4 Comparison of Mach No. distribution on different CDA and MCA 
but the exit Mach number decreases. The static pressure increases with increase in incidence angle towards positive side from $\mathrm{i}=$ 2. Keeping in view low peak suction surface Mach number and there after a gradual deceleration up to trailing end, the CDA design with -2 deg incidence ( inlet metal angle $=44$ deg ) was selected. The off design performance of original MCA profile for incidence range from $\mathrm{i}=+4$ to $\mathrm{i}=-4.947$ and of CDA profile for incidence range from $\mathrm{i}=+4$ to $\mathrm{i}=-6$ Fig. 5 produces similar results.
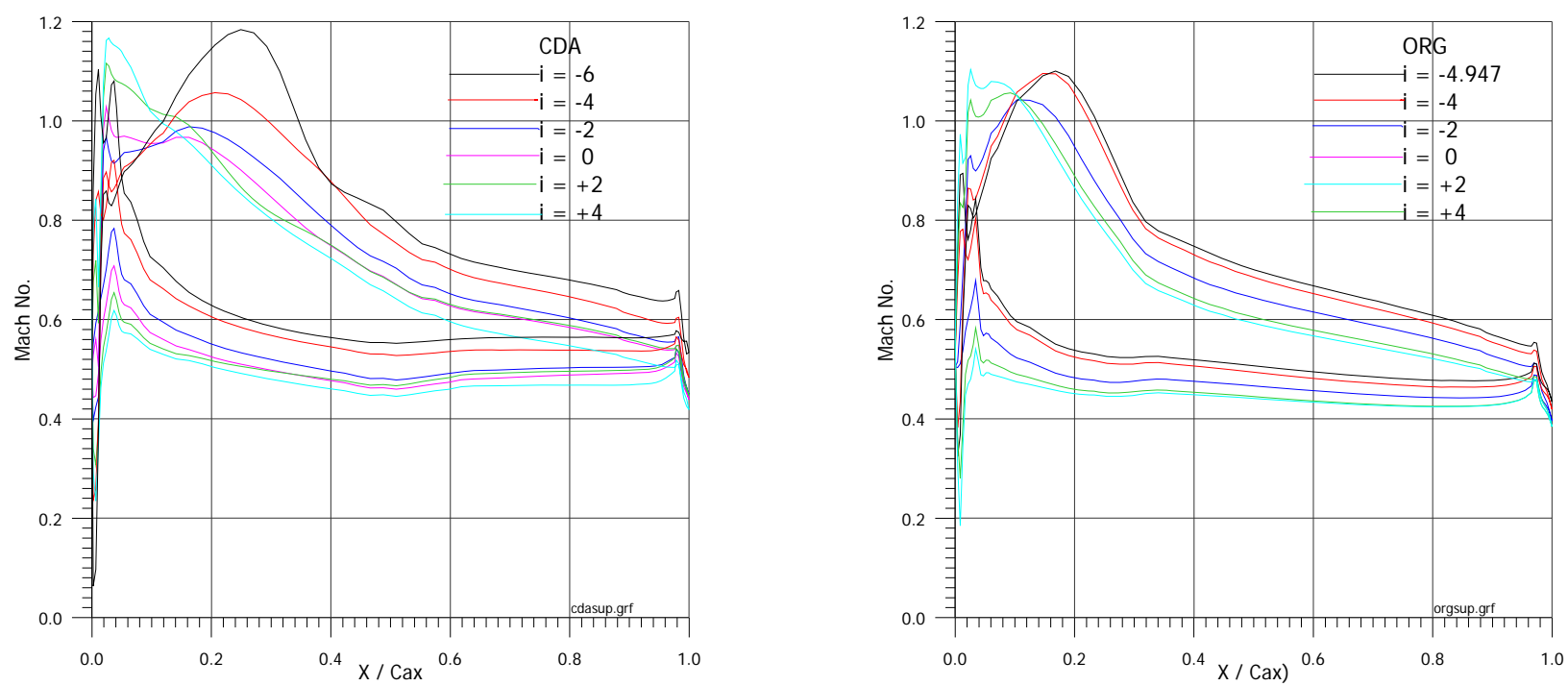

Fig. 5 Comparison of Mach number distribution for CDA and MCA at different incidence angles (off design performance)

\section{Boundary Layer Predictions for effect of variation in incidence angle}

Boundary layer computations were made for all incidence conditions for CDA and original MCA profile at design Mach number of 0.75 . The nature and variation of parameters like displacement thickness, momentum thickness and shape factor are discussed in following paragraphs.

\subsection{Displacement Thickness}

Variation of displacement thickness on both suction and pressure surfaces at design condition is shown in Fig.6 for CDA and original MCA profiles. On suction surface the displacement thickness increases gradually from leading to trailing edge for both CDA and original profile. At the trailing end, the value of displacement thickness for CDA (0.44 mm) is less than original MCA profile $(0.5 \mathrm{~mm})$. It is also observed that there is sudden decrease in value of displacement thickness at the trailing end. This is due to the cusp. Proper selection of cusp will reduce this effect.

On pressure surface there is gradual increase in the value of the displacement thickness for original profile up to trailing end but for the CDA, the displacement thickness increases gradually from leading end to about $50 \%$ of chord and then due to slight acceleration in flow or increase in Mach number, the displacement thickness growth is reduced for some distance and there after it again shows a gradual increase up to trailing end. The value for displacement thickness at trailing end for CDA (0.15 mm) is less than that for original profile $(0.17 \mathrm{~mm})$ at design incidence. As on suction surface at design condition, there is also sudden fall in value of displacement thickness at trailing end due to cusp. The results for off design performance on suction and pressure surface as shown in Fig.9a and Fig.10a indicates lower values of displacement thickness than original profile.
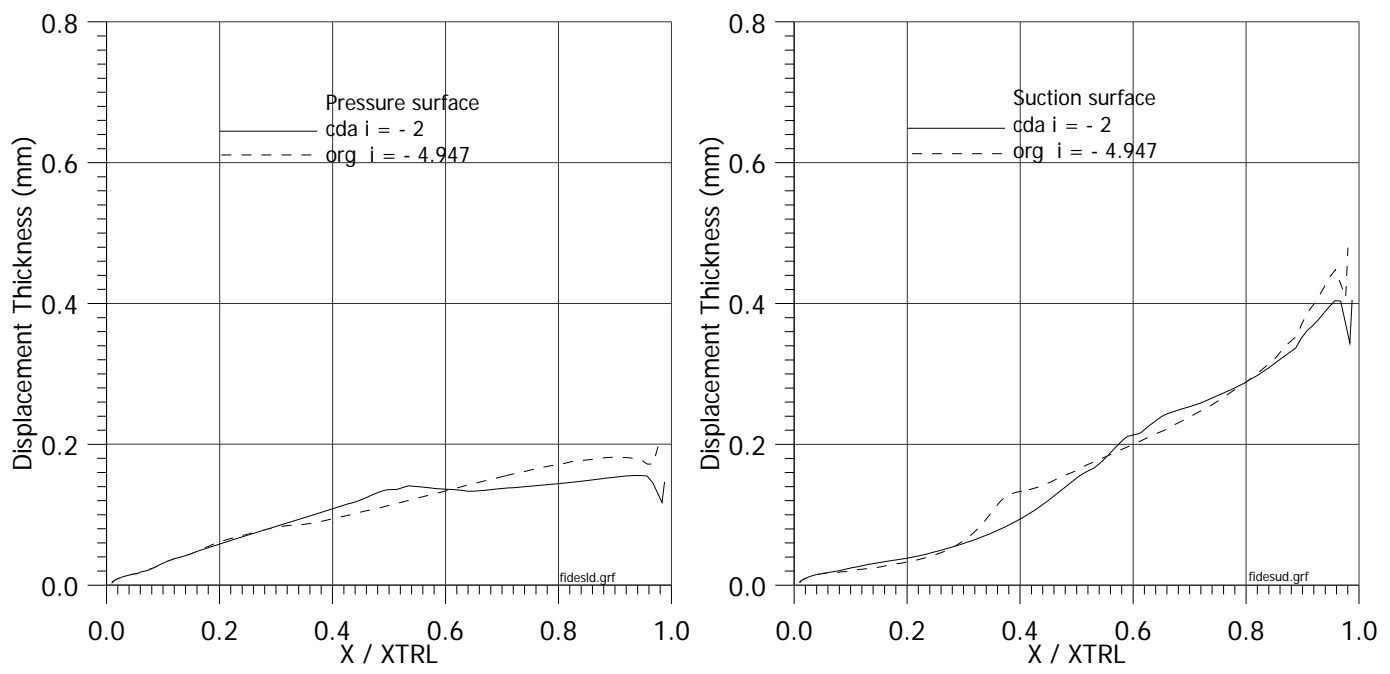

Fig. 6 Variation of Displacement Thickness on CDA and MCA 

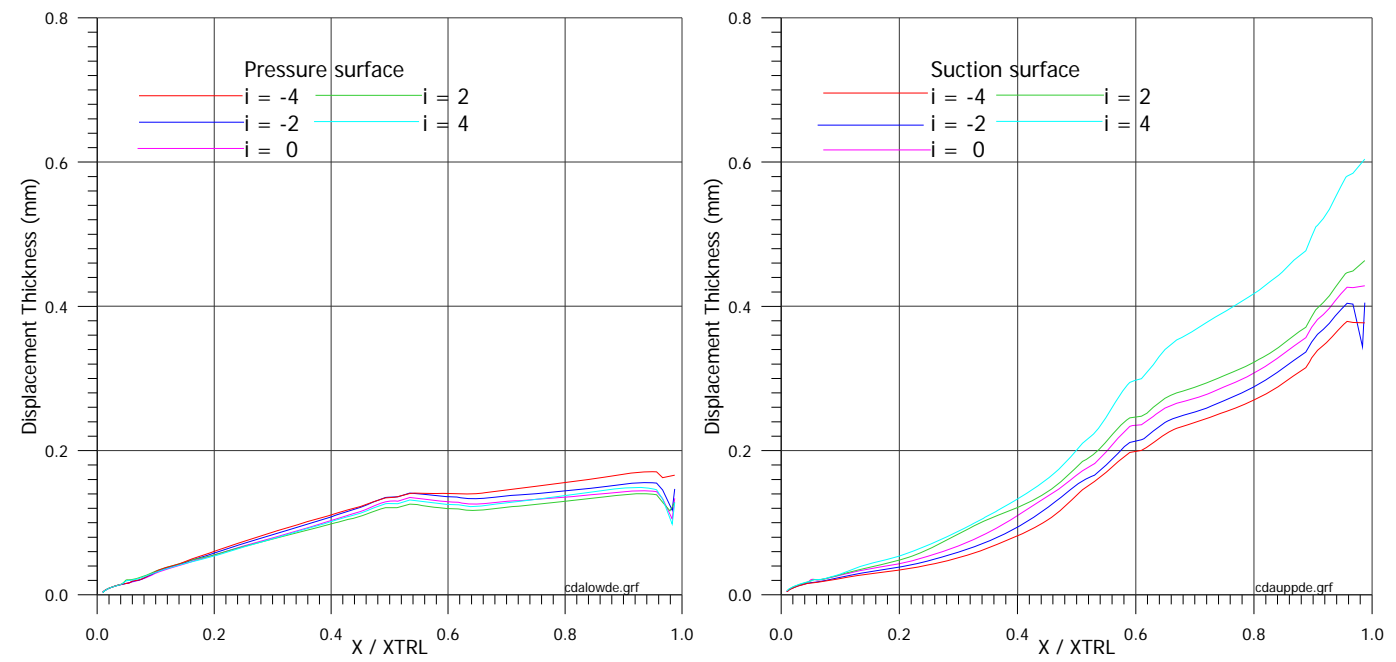

Fig. 9a Effect of variation in incidence on Displacement Thickness for CDA at 0.75 Mach No.
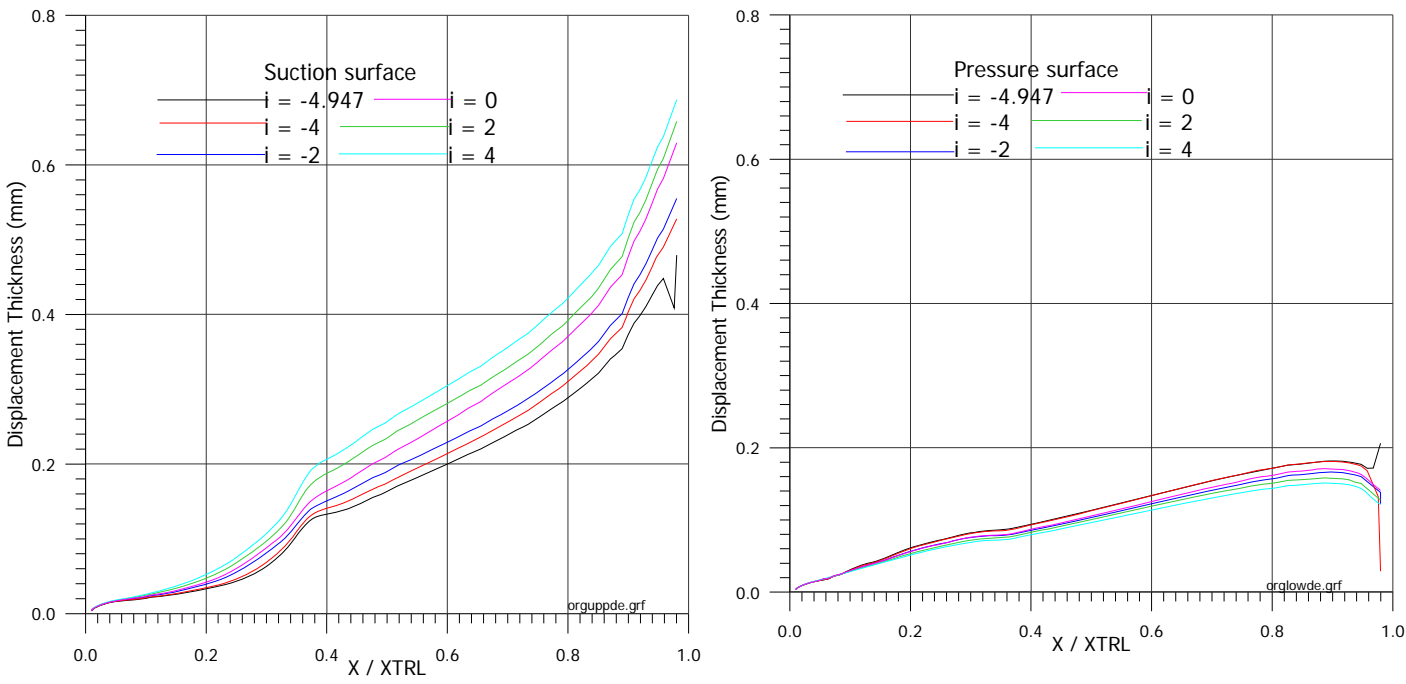

Fig. 10a Effect of variation in incidence on Displacement Thickness for original MCA at 0.75 Mach No.

\subsection{Momentum Thickness}

Variation of momentum thickness on both suction and pressure surfaces at design condition is shown in Fig.7 for CDA and original MCA profile. On suction surface the momentum thickness increases gradually from leading to trailing edge for both CDA and original profiles. At the trailing end, the value of momentum thickness for CDA $(0.253 \mathrm{~mm})$ is less than original MCA profile $(0.32 \mathrm{~mm})$. It is also observed that there is sudden decrease in value of momentum thickness at the trailing end. This is due to the cusp. Proper selection of cusp will reduce this effect.

On pressure surface there is gradual increase in the value of the momentum thickness for original profile up to trailing end but for the CDA, the momentum thickness increases gradually from leading end to about $50 \%$ of chord and then due to slight acceleration in flow or increase in Mach number, the momentum thickness growth is reduced for some distance and there after it again shows a gradual increase up to trailing end. The value for momentum thickness at trailing end for CDA (0.109 mm) is less than that for original profile $(0.118 \mathrm{~mm})$ at design incidence. As on suction surface at design condition, there is also sudden fall in value of momentum thickness at trailing end due to cusp. The results for off design performance on suction and pressure surface as shown in Fig.9b and Fig.10b indicates lower values of momentum thickness than original profile. 

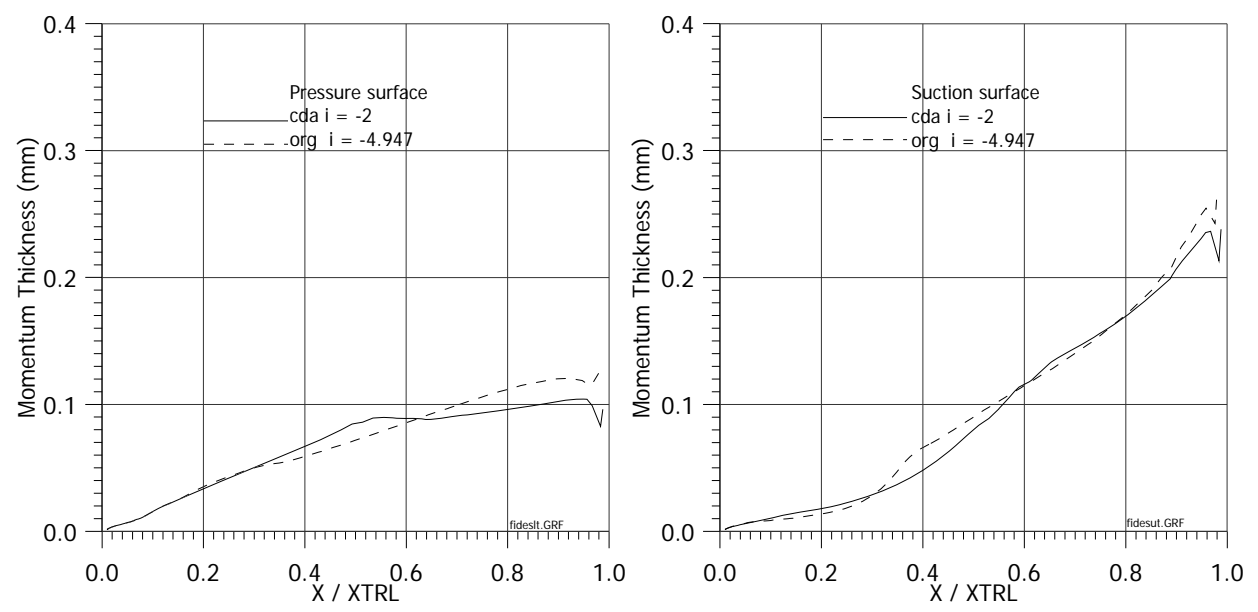

Fig.7 Variation of Momentum Thickness on CDA and MCA
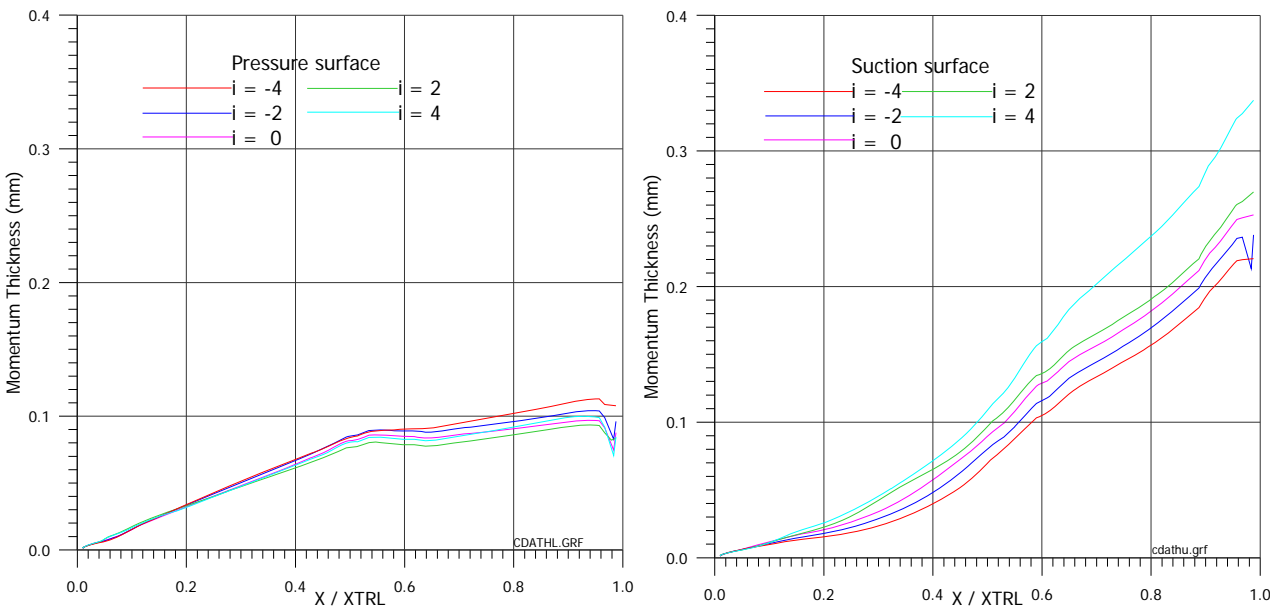

Fig. 9b Effect of variation in incidence on Momentum Thickness for CDA at 0.75 Mach No.
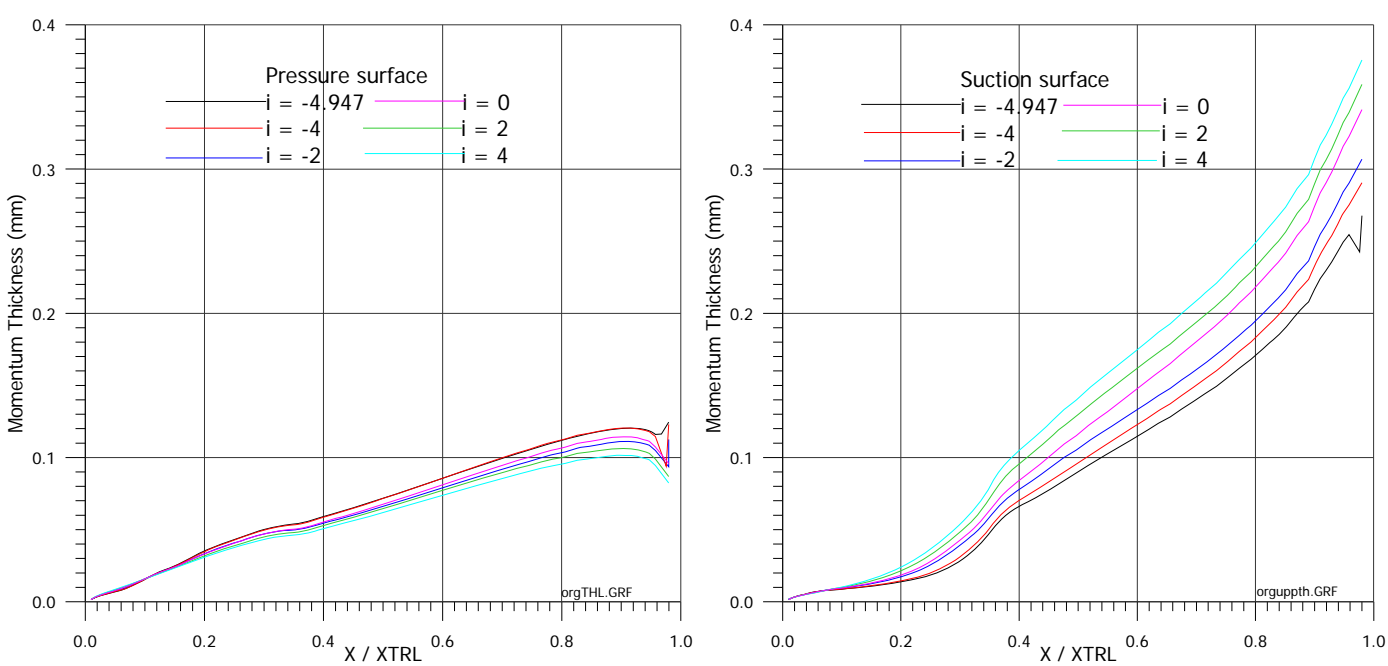

Fig. 10b Effect of variation in incidence on Momentum Thickness for original MCA at 0.75 Mach No.

\subsection{Shape Factor}

Variations of shape factor on suction and pressure surface of CDA and original MCA profiles at respective design incidence are is shown in Fig.8 The shape factor on pressure surface for both CDA and original profiles is nearly the same. Shape factor values falls gradually up to $20 \%$ chord and then remains almost constant up to trailing end. Small irregularity at the trailing end is due to cusp. Similarly on suction surface there is a sudden fall in values of shape factor for CDA up to about $10 \%$ of chord followed by gradual decrease in values up to trailing end. However, for original profile, after sudden fall in values there is rise in 
shape factor up to $20 \%$ of chord followed by uniform decrease up to trailing end. The off design performance of original MCA profile for incidence range from $i=+4$ to $i=-4.947$ and of CDA profile for incidence range from $i=+4$ to $i=-6$ showed similar results in terms of displacement thickness, momentum thickness and shape factor. The results for off design performance on suction and pressure surface as shown in Fig.9c and Fig.10c indicates lower values of shape factor than original profile.
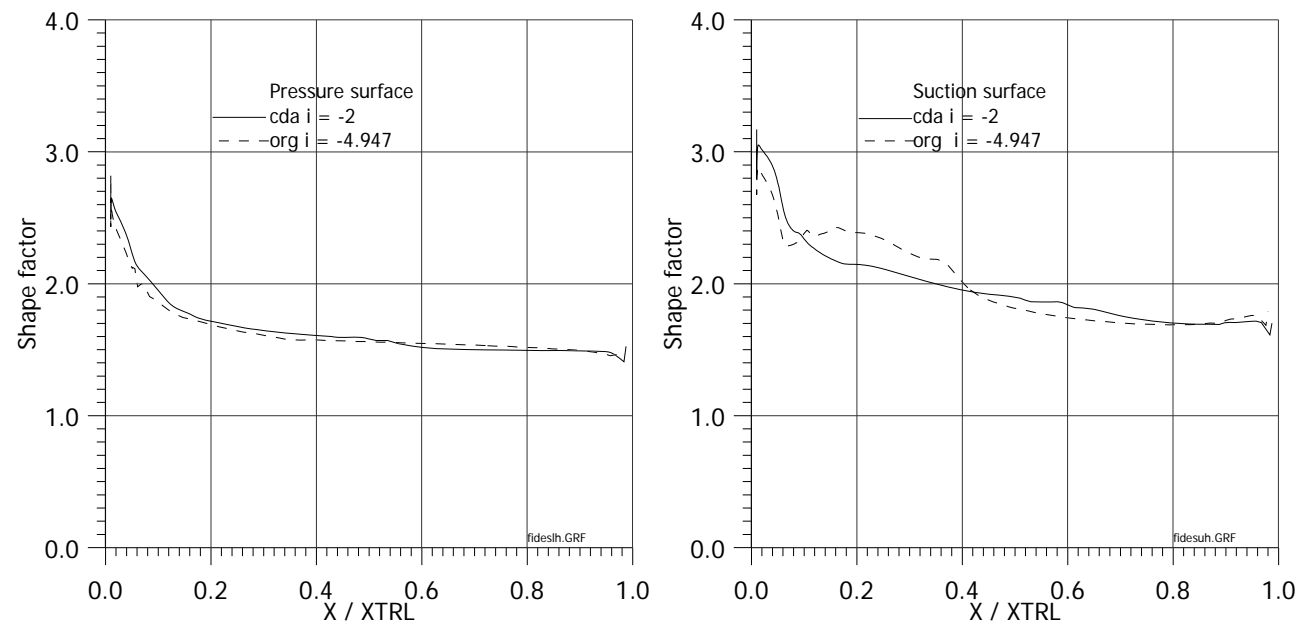

Fig. 8 Variation of Shape factor on CDA and MCA
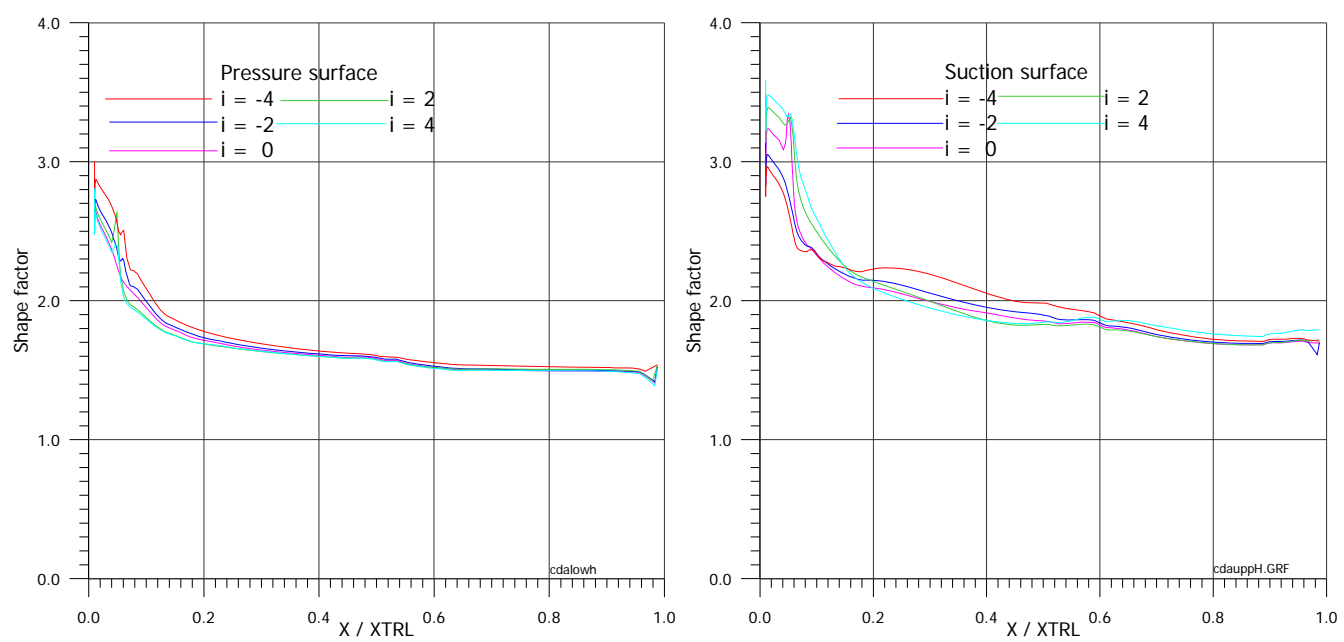

Fig. 9c Effect of variation in incidence on shape factor for CDA at 0.75 Mach No.
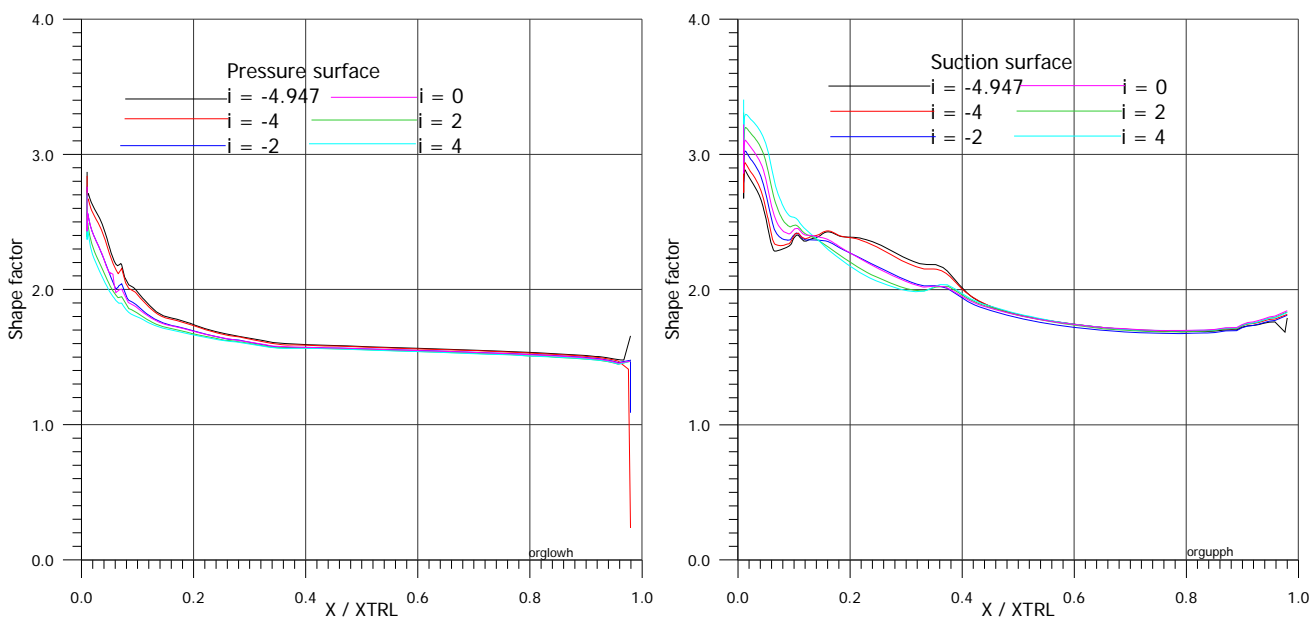

Fig. 10c Effect of variation in incidence on shape factor for original MCA Profile at 0.75 Mach No. 


\section{4 . Total Pressure Loss Coefficient}

Total pressure loss coefficient can be calculated from the correlation in Ref. (8) given as $\omega=2 *(\theta / \mathrm{c})^{*}(\sigma / \cos \beta 2)^{*}(\cos$ $\beta 1 / \cos \beta 2) 2 *\{(2 * \mathrm{H} 2 /(3 * \mathrm{H} 2-1)) /[1-(\theta / \mathrm{c}) * \sigma * \mathrm{H} 2 / \cos \beta 2]\}$

Where,

$\theta:$ : Momentum thickness in mm $\quad c:$ - Chord in $\mathrm{mm} \quad \sigma:-$ Solidity $\quad \beta 1$ : - Inlet flow angle in deg. $\beta 2:$ - Outlet flow angle in deg. H2: - Shape factor at exit of cascade.

This formula will give an approximate value of total pressure loss coefficient at the cascade exit section. When total loss is plotted against incidence angle, as shown in Fig.11, it is observed that the values increases with an increase in incidence angle towards positive side i.e. from $\mathrm{i}=-6$ to $\mathrm{i}=4$ for both CDA and original MCA profile. At respective design incidence angles for CDA and original MCA profile the value of total pressure loss coefficient for CDA (0.0144) is less than that for original MCA profile (0.162). From above discussion it is concluded that the CDA profile has better design and off design performance than the original MCA profile.

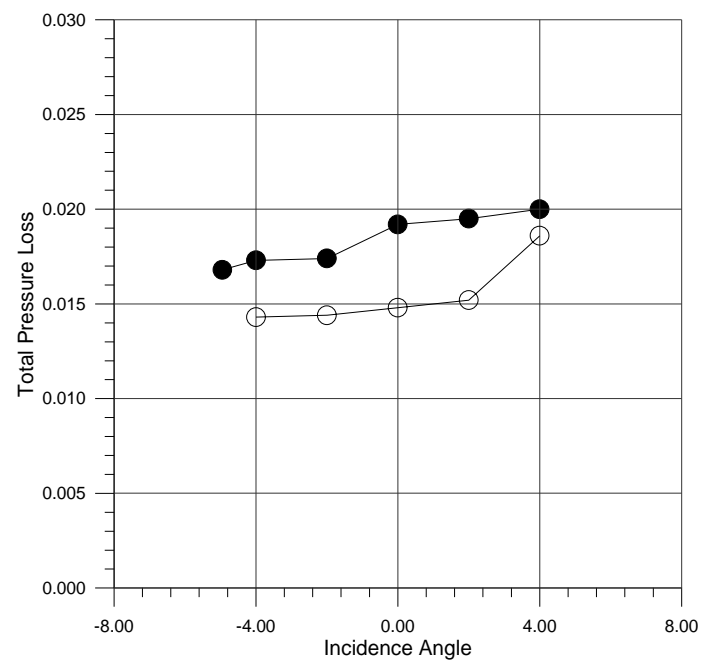

Fig. 11 Comparison of total pressure loss coefficient on CDA and MCA Profiles

\section{Effect of variation of Mach No.}

The effect of variation in inlet Mach No. shows the similar trend for CDA and Original MCA profile i.e. with the increase in inlet Mach No. the variation is too less but CDA perform better than original MCA Profile.

\section{Conclusions}

A CDA blade section has been designed equivalent to the given MCA profile. Analysis of blade elements was performed with the help of a 2-D Euler code (Denton's code) and 2-D boundary layer code. Peak suction surface Mach number for CDA section is lower than that for original MCA profile. Boundary layer parameters $\left(\delta^{*}, \theta\right)$ have lower values for CDA than that for original MCA profile. Total pressure loss for CDA is lower than that for original MCA profile.

\section{Nomenclatures}

$\beta_{1}{ }^{*}$-- Inlet Metal angle (deg)

$\delta$-- Boundary layer thickness (mm)

$\alpha$, ALPA -- Alpa chord angle (deg)

$\mu \mathrm{T} \quad--$ Turbulent viscosity $\left(\mathrm{N} \mathrm{s} / \mathrm{m}^{2}\right)$

$\tau$, Tow, s -- Pitch (mm) $\delta$, DEAN -- Deviation angle (deg)

$\delta^{*}$-- Displacement thickness (mm)

$\theta \quad$-- Momentum thickness (mm)

$\mu \mathrm{L} \quad$-- Laminar viscosity $\left(\mathrm{N} \mathrm{s} / \mathrm{m}^{2}\right)$ 


\section{References}

[1] Whitcomb, R. T. and Clark, L. R., 1965, “An aerofoil shape for efficient flight at supercritical Mach numbers,” NASA TMX 1109.

[2] Bauer, F., Garabedian, P., Korn, D., 1972, "Supercritical Wing Sections, Lecture notes In Economics And Mathematical Systems,” Vol. 66, Springer - Verlag, New York.

[3] Bauer, F., Garabedian, P., Korn, D., 1977, “Supercritical Wing Sections III,” Lecture notes in Economics And Mathematical Systems, Vol. 150, Springer - Verlay, NewYork.

[4] Lubenstein. J. H., Robideu B. A. Ross A. K, 1984, “Aerofoil Shape For Arrays Of Aerfoils ,” U. S. Patent 4,431,376.

[5] Q. H. Nagpurwala, S.A.Guruprasad, H. Girigoswamy, B. R. Pai, "Experimental and Analytical Studies of A Transonic Stator having Controlled Diffusion Aerofoil Sections with End Bends,” Propulsion Division, National Aerospace Laboratories , Bangalore - 560017 ,ISABE 95-7074.

[6]Q. H. Nagpurwala, B. R. Pai, S. A. Guruprasad, Characteristics of conventional and controlled diffusion stator blades in a transonic compressor stage, Propulsion Division, National Aerospace Laboratories, Bangalore - 560017 ISABE, 93-7124.

[7] Horlock, J. H., 1958, “Axial Flow Compressors,” Butteworth.

8] M. Anbarasu, B. R. Pai, “A Method for Designing Transonic Compressor Blade Profiles,Propulsion Division,” National Aerospace Laboratories , Bangalore - 560017 PDPR9016. 